\title{
EMPA and innovations
}

\author{
H. W. Fritz, Head of Division Building Materials \\ Suriss Federal Laboratories for Materials Testing and Research (EMPA), CH-8600 Diblendorf, Switzerland
}

EMPA is an interdisciplinary institute providing, as the name implies, both research facilities and routine testing to comply with a range of industrial, environmental and official governmental requirements. Some specific practice-oriented current research projects are described which exemplify many of EMPA's capabilities.

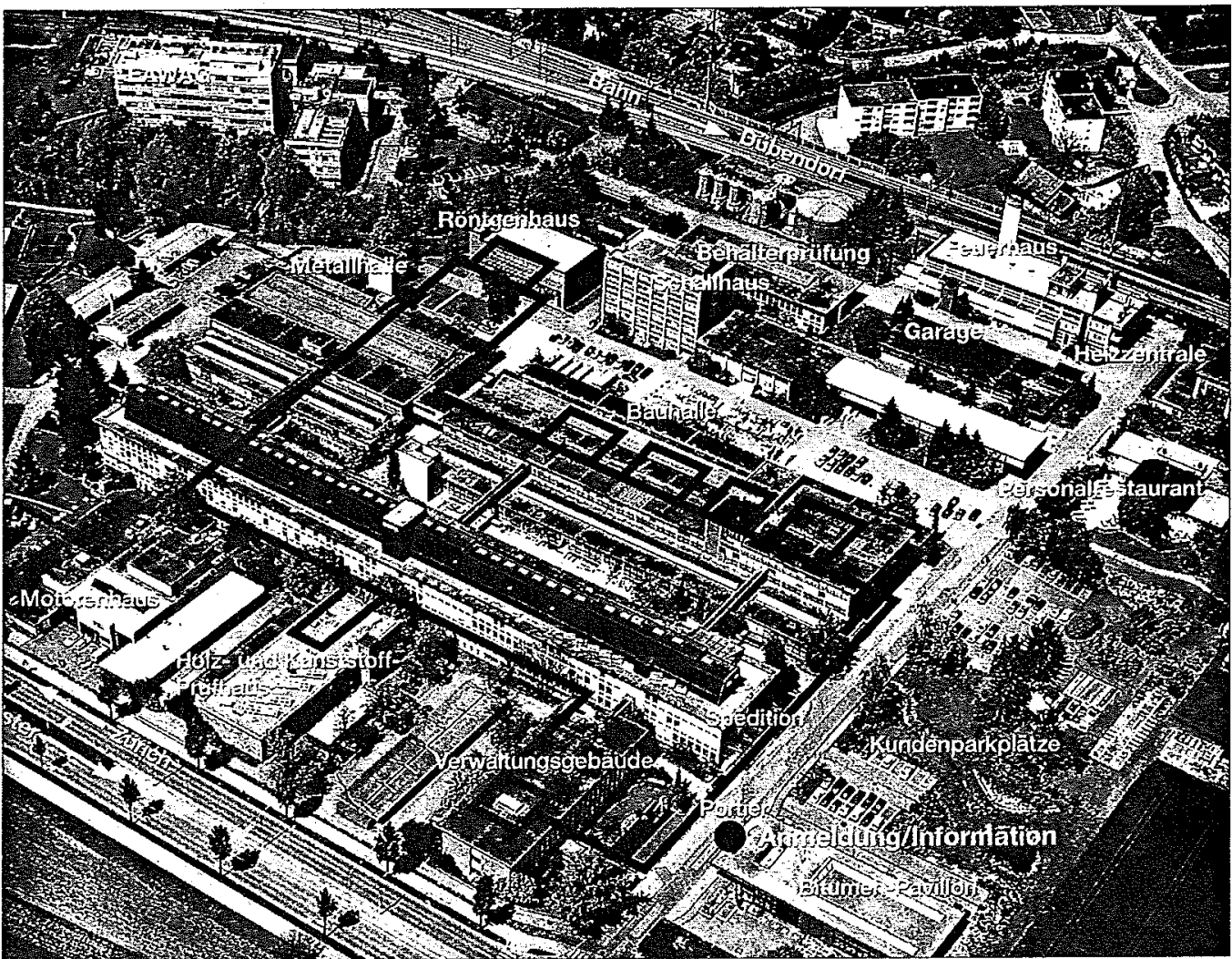

Fig. 1.

\section{BACKGROUND}

EMPA was founded in 1880 under the leadership of Prof. L. v. Tetmajer and was originally called "Institute for the Testing of Building Materials". At that time, EMPA was situated in the heart of Zurich, in the basement of the Swiss Federal Institute of Technology, ETHZ. It was already recognized then that the study of the properties of materials and their evaluation by a neutral body were indispensable. This basic goal has remained valid for EMPA up to the present. However, in the meantime, its fields of activity have expanded considerably. EMPA has always adjusted its role not only in society, industry and science, but also according to the rapid development of engineering and technology and to ecological problems and advances for European integration.

The strategy of 1988 led to a shift of emphasis which was intensified by the new EMPA regulation of January 13, 1993. Applied research and development as well as consulting services were thus favoured at the expense of less synergetic routine testing.

From a handful of employees in 1880, EMPA has grown into an establishment of approximately 775 collaborators, 541 of whom work in Dübendorf, some 30 in Thun and 204 in St. Gall. The aerial view in Fig. 1 shows the premises of EMPA Dübendorf, inaugurated in 1962. Fig. 2 shows its largest testing hall where the structure section can test large elements. 




Fig. 2.

\section{MAIN ACTIVITIES}

EMPA is dedicated mainly to promoting safety for man and the environment. Furthermore, EMPA supports projects that strengthen the Swiss economy. All activities are carried out in a strictly neutral manner.

The spectrum of EMPA's activities may be broadly divided into three main areas:

- building materials,

- environmental technology, and

- safety and quality (of structures, installations, products and procedures).

EMPA strongly emphasizes collaboration amongst the different disciplines. EMPA supports the technical universities and other educational institutes with its teaching activities and research. In addition, training and furthereducational courses are given in the various technical areas.

EMPA is involved in the following fields:

- research and development, above all with the goals of improving the economic and ecological use of materials as

Il as the development of test methods and equipment,

- official tests and consulting,

- collaboration in the preparation of standards and regulations in the respective technical areas,

- participation in the enforcement of federal ordinances.
Thanks to the development of an exemplary and comprehensive quality assurance system, EMPA has established the prerequisites for accreditation. A number of areas have been accredited in accordance with EN 45001. Agreements with foreign partner institutes ensure that tests performed at EMPA within the framework of subcontracting are also accepted by European Union countries.

EMPA has achieved world-wide leadership and recognition for its pioneering work in building materials, applying carbon fibers in cables to replace steel in suspension bridges, perfecting systems to use carbon fiber reinforced epoxy composites and special epoxy adhesives to rehabilitate deteriorated bridges.

Its mission is specialised testing and research in the fields of materials, environmental technology, safery and qualitiy of systems, building construction, installations, processes and products. EMPA is the national resource centre committed to safety and quality of human life with a vision of economic, ecologically balanced and sustainable development. EMPA fulfils its mission by maintaining core skills, developing and advancing innovative technologies and achieving a competitive and leadership role.

\section{RILEM AND EMPA}

Since the foundation of RILEM, the International Union of Testing and Research Laboratories for Materials and Structures, EMPA has actively contributed in many fields and functions within the RILEM family. As the 50th anniversary of RILEM was celebrated during the 51 st annual meeting at the premises of ETHZ near the end of September 1997, we seized the opportunity to invite the participants to visit the EMPA in Dübendorf near Zürich. This enabled us to present some lighlights of our activities. The visitors, three groups of about twelve participants, were guided from highlight to highlight, according to the marked route in Fig. 1.

The interested listeners were given specific information related to innovations in the field of CFRP in civil engineering. Director Prof. U. Meier explained a practiceoriented research project aimed at replacing conventional and prestressing steel reinforcement of centrifugally cast concrete poles (Fig. 3). In Fig. 4, C. A. M. de Smet pre-

\section{MANAGEMENT, ORGANISATION}

EMPA is a neutral and objective organization operating according to commercial principles. EMPA is divided into two research areas with locations in Dübendorf, St. Gall and Thun. It is managed by a four-person board made up of the EMPA president, the two directors of the testing and research areas and the head of the technical area resources. EMPA comprises a total of 30 technical sections, each having its special areas of interest. The technical areas of logistics, controlling and marketing are undertaken in common for the entire EMPA.

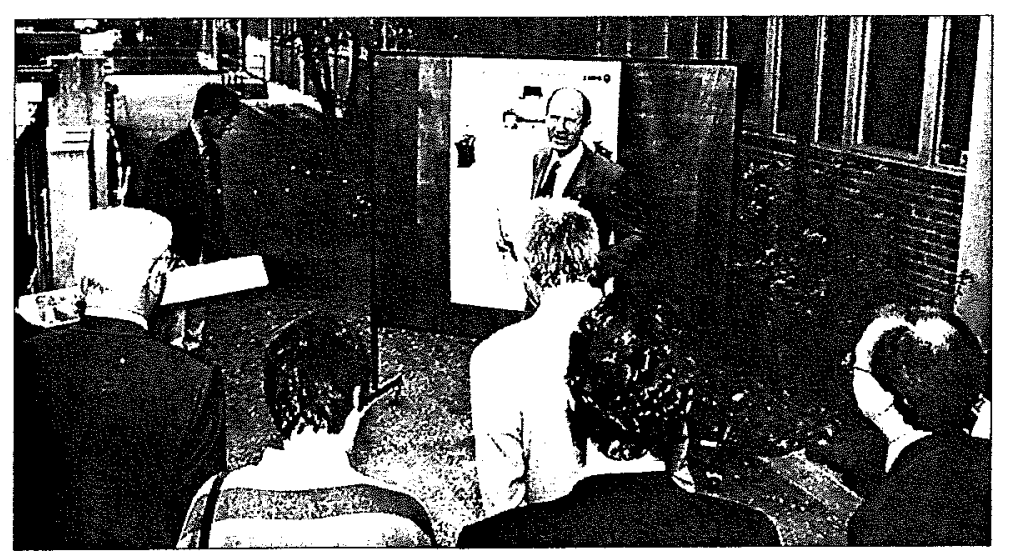

Fig. 3. 


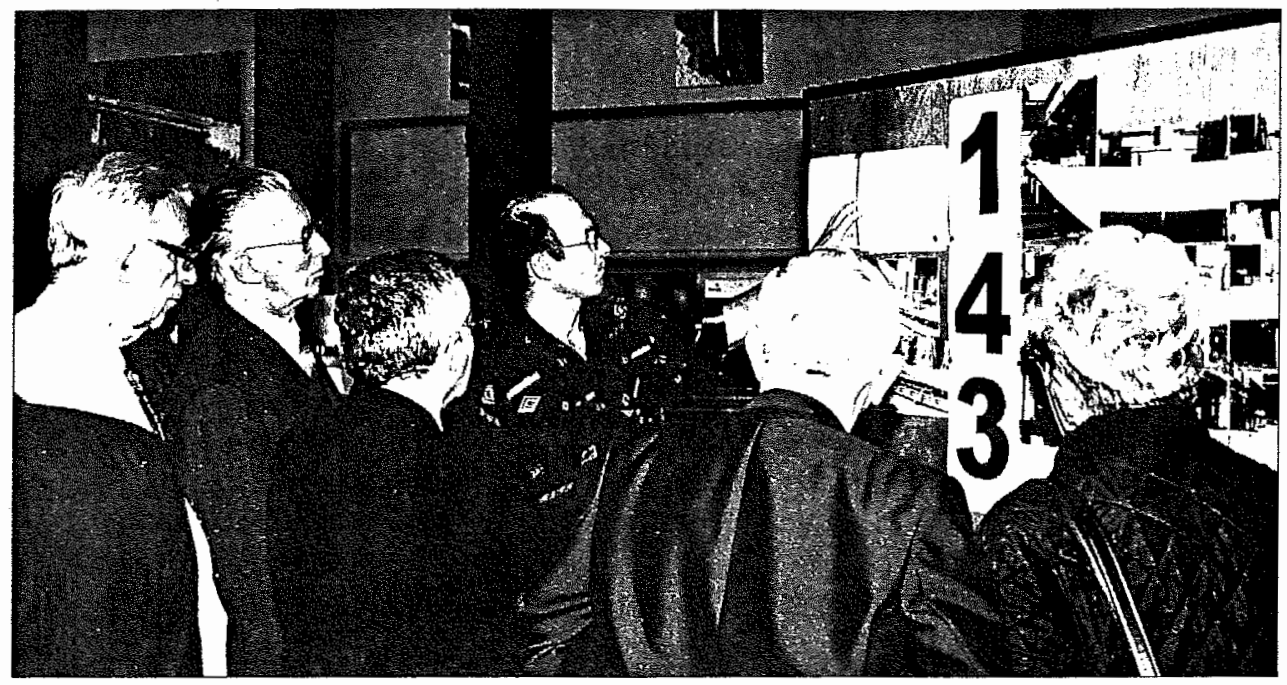

Fig. 4.

sents main findings from another practice-oriented research project. In a first part of the project, carried out until 1995, the feasability of using CFRP tendons for statically determinate systems was proven. The main results of the second part, dealing with two-span girders, show that CFRP strands can be used to prestress concrete for statically indeterminate systems too.

\section{INNOVATIVE RESEARCH}

The following seven contributions are examples of recent and innovative practice-oriented research projects being carried out at EMPA in the field of building, structures, materials, testing and measurement techniques:
- "Prestressed concrete using carbon fibre reinforced plastic (CFRP) strands",

A. Maissen, C.A.M. de Smet

- "Characterization of unidirectional carbon fibre reinforced-laminates",

by M. Motavalli, P. Flüeler

- "Application of Experimental Modal Analysis in practice",

C. Krämer, C.A.M. de Smet

- "Use of TBM chips as concrete aggregate", Hp. Olbrecht, W. Studer

- "Infrared thermography", T. Lüthi

- "Ventilation studies in a room climate laboratory", Th. Rüegg, A. Weber

- "Ground Penetrating Radar for road engineering", J. Hugenschmidt. 